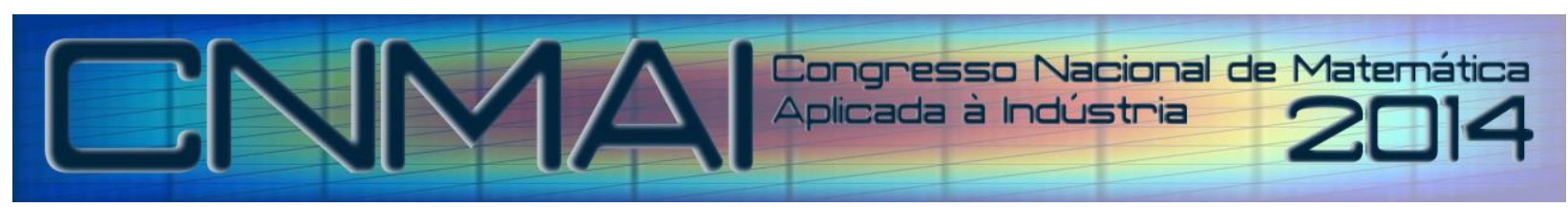

18 a 21 de novembro de 2014, Caldas Novas - Goiás

\title{
SIMULAÇÃO DA TRANSFERÊNCIA DE CALOR TRANSIENTE UTILIZANDO O MÉTODO DOS ELEMENTOS DE CONTORNO
}

\author{
Rafael Marques Campos, rmarquescampos@gmail.com ${ }^{1}$ \\ ${ }^{1}$ Universidade de Brasília - Departamento de Engenharia Mecatrônica ... Campus Universitário Darcy Ribeiro, \\ Faculdade de Tecnologia, Asa Norte, Brasília
}

\begin{abstract}
Resumo: Este trabalho apresenta o desenvolvimento de programas com a finalidade de simular numericamente a transferência de calor no processo de prototipagem rápida por deposição de metal em camadas sucessivas utilizando soldagem a arco, também chamada de soldagem $3 D$ (3D Welding). Com o objetivo de conseguir prever o comportamento do material depositado, a transferência de calor é analisada. Um modelo numérico que utiliza o método dos elementos de contorno é desenvolvido, a partir do qual se torna possível simular determinadas condições. Para as simulações são consideradas condições de contorno de temperatura, fluxo de calor, convecção e radiação, sendo possível também o uso de fontes pontuais de calor. Dentro do método dos elementos de contorno são utilizadas duas metodologias, o método da reciprocidade dual e a solução fundamental transiente. Ao final valida-se o código comparando os resultados numéricos com soluções analíticas.
\end{abstract}

Palavras-chave: elementos de contorno, transferência de calor, reciprocidade dual, solução fundamental

\section{INTRODUÇÃ̃O}

Ultimamente, os processos de prototipagem rápida (em inglês, Rapid Prototyping) vem ganhando força na indústria. Isso devido à contínua exigência de minimizar gastos financeiros e de tempo na fabricação de protótipos. Comparado a outros processos de fabricação, a construção camada a camada dá maior liberdade na confecção da peça, sendo esta a vantagem que torna o método tão atrativo.

Na soldagem 3D, o processo começa com a criação de um modelo sólido em CAD (Computer Aided Design). Então é usado um software para traçar o percurso da tocha, o modelo é dividido em camadas sucessivas, traçando um caminho contínuo que será percorrido pelo robô de solda. Já que o metal de solda sai a altas temperaturas e é um bom acumulador de calor, o tempo de resfriamento da poça de solda aumenta gradativamente, com isso a altura esperada de cada camada varia.

O acúmulo de calor gera, por consequência, variações das características do sólido. Por isso é necessário um estudo mais aprofundado da transferência de calor com objetivo de controlar as variáveis pertinentes para melhorar a qualidade do protótipo.

O intuito deste trabalho é criar um programa, através do software MatLab, capaz de simular numericamente problemas de transferência de calor com diversas condições de contorno. O método no qual a pesquisa se baseia é o método dos elementos de contorno (MEC) e tem como objetivo criar programas capazes de simular problemas de transferência de calor com diferentes condições de contorno, para que seja possível simular casos de soldagem 3D usando um robô de solda MIG/MAG.

\section{MÉTODO DOS ELEMENTOS DE CONTORNO}

O método dos elementos de contorno (BEM - Boundary Elements Method) é uma técnica computacional para a aproximação de soluções de problemas na mecânica do contínuo. Esses problemas são caracterizados matematicamente por equações diferenciais governantes cujas soluções analíticas, dependendo da complexidade do caso, não são possíveis de encontrar.

Técnicas de simulação numérica podem ser divididas em três grandes vertentes: diferenças finitas, elementos finitos e elementos de contorno. Todos os três envolvem Substituição de expressões envolvendo cálculo por relações algébricas 
aproximadas, uso de malha computacional para descrever a geometria do corpo a ser simulado e solução de equações algébricas para determinar as condições desconhecidas na malha.

Nenhum método se mostra superior aos outros em todos os casos. Cada um possui suas vantagens e desvantagens. No caso dos elementos de contorno algumas de suas vantagens são: simples modelagem de fronteiras e condições de contorno, necessita apenas de malha superficial, ideal para problemas com domínio infinito e a precisão da solução não está ligada à quantidade de pontos internos. Em contrapartida é necessária uma solução fundamental que represente o comportamento do fenômeno físico, possui uma grande quantidade de integrações numéricas e funções complexas e tem matrizes cheias e assimétricas.

\subsection{Transferência de Calor}

A lei de condução de calor de Fourrier é determinada experimentalmente e relaciona o gradiente de temperatura com a taxa de condução de calor no tempo. A partir dessa lei são encontradas as equações de condução de calor, que para uma parede extensa plana com condutividade constante é representada pela Eq. (1) quando em regime permanente e pela Eq. (2) quando em regime transiente como mostra Çengel (2008).

$$
\begin{gathered}
\nabla^{2} T=0 \\
\nabla^{2} T-\frac{1}{\alpha} \frac{\partial T}{\partial t}=0
\end{gathered}
$$

Onde $\nabla^{2}$ é o Laplaciano, $T$ é a temperatura, $t$ é o tempo e $\partial$ é o indicador de derivação parcial.

Existem quatro condições de contorno principais: temperatura conhecida, fluxo conhecido, convecção e radiação. As equações gerais da condução de calor não incorporam nenhuma informação sobre as condições de contorno, necessitando então determinar essas condições térmicas nas superfícies das fronteiras para que a descrição do problema esteja completa.

As condições de temperatura e fluxo são mais simples, onde se tem diretamente ou o valor da temperatura ou o do fluxo de calor. A condição de contorno de convecção é um pouco mais complexa, porém o fluxo varia linearmente com a temperatura, considerando o coeficiente de transferência de calor por convecção constante. Por último, a condição de contorno de radiação é a mais complicada, já que o fluxo de calor varia com a temperatura de forma não linear. A radiação considerada é completamente dissipada para o meio externo, fazendo com que o fator de forma (ou fator de visão) seja sempre unitário.

\subsection{Solução Fundamental}

Uma das principais características do método dos elementos de contorno é o uso de soluções conhecidas do problema a ser investigado. Na condução de calor, a resposta térmica estacionária de um meio infinito condutor para um ponto fonte com geração interna de energia é chamada solução fundamental. Essa solução deve satisfazer a equação diferencial dada pela Eq. (3), como mostram vários trabalhos (Brebbia, 1992; Partrdge, Brebbia e Wrobel, 1991 e Kane, 1994).

$$
\nabla^{2} T^{*}=-\frac{\delta(x-d)}{k}
$$

Onde $T^{*}$ é a solução fundamental de temperatura, $\delta(x-d)$ é o delta de Dirac que tende ao infinito quando o ponto analisado $(x)$ estiver sobre o ponto fonte $(d)$ e $k$ é a condutividade térmica do material.

A função delta de Dirac é ideal para representar cargas e fontes pontuais, e é ativada quando o ponto fonte coincide com o ponto campo. Intuitivamente o problema parece artificial, já que a temperatura no centro da fonte concentrada deve ser infinita, certamente o material derreteria e em seguida se vaporizaria. Matematicamente, é dito que esse comportamento é singular, e esse é um ponto de singularidade. Ou seja, para a compreensão do método, a melhor maneira para caracterizar esse tipo de função é admitir que ela é fisicamente estranha mas matematicamente útil.

Para estudar a solução da Eq. (3), considere a origem do sistema de coordenadas bidimensional o ponto fonte $(d=0)$ assim como mostra a Fig. 1, adaptada do trabalho de Kane (1994). Essa mesma equação diz que o Laplaciano da distribuição de temperatura é zero em todos os pontos, exceto na origem. Portanto, é necessário selecionar uma equação fundamental que tenha um comportamento singular na origem. Outra consideração é que o problema deve ter simetria cilíndrica com relação à origem, ou seja, os pontos campo a uma distância radial $R$ são equivalentes.

Com todas essas considerações para o caso bidimensional, a física do problema sugere que a temperatura se comporte da forma $T=\ln (R)$, já que o fluxo de calor, $\left(\frac{d T}{d R}\right)$, deve se comporta como $\frac{1}{R}$ no caso bidimensional. As soluções fundamentais para o caso permanente e bidimensional estão representadas nas Eq. (4) e Eq.(5). 


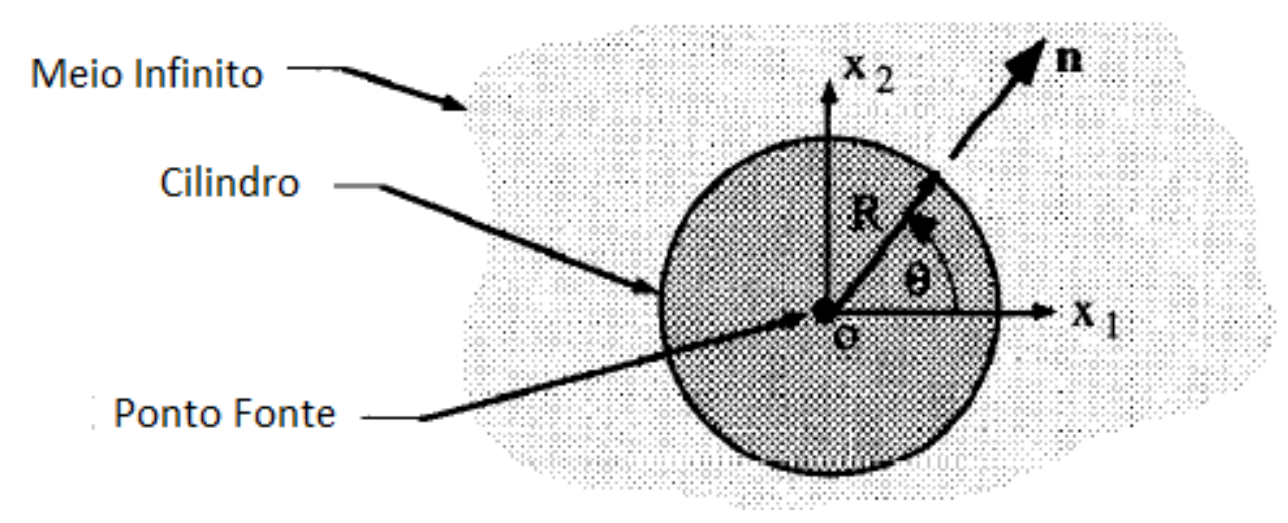

Figura 1. Representação do ponto fonte no domínio.

$$
\begin{gathered}
T^{*}=-\frac{1}{2 \pi k E} \ln (R) \\
\overrightarrow{q^{*}}=-\frac{1}{2 \pi k E}\left[\left(x_{i}-x_{d}\right) \overrightarrow{e_{1}}+\left(y_{i}-y_{d}\right) \overrightarrow{e_{2}}\right]
\end{gathered}
$$

Onde $E$ é a espessura do elemento, considerada unitária, $x$ e $y$ são as coordenadas x e y, $e_{j}$ é o vetor unitário na direção $\mathrm{j}$ e os índices $i$ e $d$ representam o ponto campo e o ponto fonte, respectivamente.

A primeira afirmação que pode ser feita para o caso tridimensional é o fato de que a energia conduzida através da esfera deve ser igual à energia gerada no seu interior. Para afirmar isso, a energia atravessando a superfície deve se comportar como $R^{-2}$, já que a área do elemento agora se comporta como $R^{2}$ pois o problema se torna esférico. A partir daí, infere-se que a solução fundamental de temperatura se comporte como $R^{-1}$. Com isso, as soluções fundamentais encontradas que satisfazem a Eq. (3) são as Eq. (6) e Eq. (7).

$$
\begin{gathered}
T^{*}=-\frac{1}{4 \pi k} R^{-1} \\
\overrightarrow{q^{*}}=-\frac{1}{4 \pi} R^{-3}\left[\left(x_{i}-x_{d}\right) \overrightarrow{e_{1}}+\left(y_{i}-y_{d}\right) \overrightarrow{e_{2}}+\left(z_{i}-z_{d}\right) \overrightarrow{e_{3}}\right]
\end{gathered}
$$

A solução fundamental dependente do tempo para problemas de transferência de calor e sua derivada estão representadas pelas Eqs. (8) e (9), como mostra Effren (1997).

$$
\begin{gathered}
T^{*}=-\frac{1}{4 \pi \tau \alpha} \exp \left(-\frac{R^{2}}{4 \alpha \tau}\right) H e(\tau) \\
\overrightarrow{q^{*}}=-\frac{R \frac{\partial R}{\partial n}}{8 \pi \tau^{2} \alpha^{2}} \exp \left(-\frac{R^{2}}{4 \alpha \tau}\right) H e(\tau)
\end{gathered}
$$

Onde $R \frac{\partial R}{\partial n}$ é a projeção da distância $\mathrm{R}$ sobre a normal do contorno, $\tau$ é o tempo de observação subtraído o tempo de aplicação da fonte $\mathrm{He}(\tau)$ chama-se função de Heaviside, que enfatiza que não pode haver resposta antes de ocorrer a aplicação da fonte pontual.

\subsection{Equação Integral}

Aplicando a segunda identidade de Green com a solução fundamental da temperatura e a resposta real da temperatura do problema tem-se a Eq. (10), como mostra Kane (1994).

$$
\int_{\Omega} \nabla^{2} T T^{*} d \Omega-\int_{\Omega} \nabla^{2} T^{*} T d \Omega=\int_{S} T^{*} \vec{\nabla} T \cdot \vec{n} d S-\int_{\mathrm{S}} T \vec{\nabla} T^{*} \cdot \vec{n} d S
$$

Onde $\Omega$ representa o domínio, $S$ o contorno e $\vec{n}$ é o vetor normal à superfície.

A partir das propriedades da função delta de Dirac e sabendo que para problemas permanentes o primeiro termo é zero, como mostrado na Eq. (1), obtém-se as equações integrais para o caso permanente, Eq. (11), e para o caso transiente a Eq. (12). Tanto para o caso bidimensional quanto para o caso tridimensional a equação integral é a mesma. $O$ fator 
geométrico $(c)$ é 1 quando o ponto fonte está dentro do contorno, 0 quando está fora do contorno e 0,5 quando o ponto fonte estiver no contorno, e este contorno for suave.

$$
\begin{gathered}
c T(d)=\int_{S} T q^{*} \cdot \vec{n} d S-\int_{S} T^{*} q \cdot \vec{n} d S \\
c T=\alpha \int_{t_{0}}^{t_{f}} \int_{S} T^{*} q d S d t-\alpha \int_{t_{0}}^{t_{f}} \int_{S} q^{*} T d S d t
\end{gathered}
$$

\subsection{Método da Reciprocidade Dual}

O método da reciprocidade dual tem como função transformar integrais de domínio em integrais de contorno. Seu uso é vasto na solução de problemas transientes e é muito simples se comparado a outros métodos, dado a sua abrangência. O método da reciprocidade dual propõe usar uma série de soluções particulares, ao invés de apenas uma única função. Isso porque em geral é difícil encontrar uma solução que satisfaça a Eq. (13), equação de Poisson, principalmente por serem casos não lineares e que dependem do tempo.

$$
\nabla^{2} T=b
$$

Onde b é uma função que pode depender da posição, do potencial ou do tempo. A solução deste problema é composta da soma da solução particular com solução da homogênea, Eq. (14).

$$
T=\widehat{T}+\tilde{T}
$$

Sendo $\widehat{T}$ a solução particular e $\widetilde{T}$ a solução homogênea é possível escrever a Eq. (15).

$$
\nabla^{2} \widehat{T}=b
$$

O método da reciprocidade dual propõe usar uma série de soluções particulares, $\widehat{T}_{j}$, ao invés de apenas uma única função $\widehat{T}$. Isso porque em geral é difícil encontrar uma solução que satisfaça a Eq. (15) principalmente em casos não lineares e que dependem do tempo. O número de soluções $\widehat{T}_{j}$ encontradas é igual ao número total de nós do problema, somando os nós no contorno $(N)$ e também os pontos internos $(L)$.

Com isso, a Eq. (16) é proposta, como mostra Partridge, Brebbia e Wrobel (1991):

$$
b \cong \sum_{j=1}^{N+L} \alpha_{j}\left\{f_{j}\right\}
$$

onde $\alpha_{j}$ são coeficientes desconhecidos inicialmente e $f_{j}$ são funções de aproximação. A solução particular e a função de aproximação são relacionadas segundo Eq. (17).

$$
\nabla^{2} \widehat{T}_{j}=\left\{f_{j}\right\}
$$

A função de aproximação $f_{j}$ depende da geometria do problema. Existem várias funções de aproximação possíveis, escolher a mais apropriada faz parte da solução do problema.

Para obter a equação que rege o método são necessárias algumas manipulações matemáticas. Substituindo a Eq. (17) na Eq. (16) encontra-se uma equação que define o vetor $b$. Aplicando esse valor na Eq. (13) obtém-se a Eq. (18).

$$
\nabla^{2} T=\sum_{j=1}^{N+L} \alpha_{j}\left(\nabla^{2} \widehat{T}_{j}\right)
$$

Manipulando matematicamente, obtém-se a equação integral do método, representada pela Eq. (19).

$$
c_{i} T_{i}+\int_{\Gamma} q^{*} T d \Gamma-\int_{\Gamma} T^{*} q d \Gamma=\sum_{j=1}^{N+L} \alpha_{j}\left(c_{i} \widehat{T}_{i j}+\int_{\Gamma} q^{*} \widehat{T}_{j} d \Gamma-\int_{\Gamma} T^{*} \hat{q}_{j} d \Gamma\right)
$$

Utilizando um método de integração no tempo é possível resolve a Eq. (2), que é uma equação de Poisson, e simular problemas de transferência de calor transiente. 


\section{RESULTADOS}

\subsection{Programa Permanente 2D}

Para a validação simulou-se o problema descrito pela Fig. 2, presente no livro "the dual reciprocity boundary element method" de Partridge, Brebbia e Wrobel (1991). Nesse exercício uma das condições de contorno é a convecção, com um coeficiente $h=10 \mathrm{~W} / \mathrm{m}^{2 \circ} \mathrm{C}$ e temperatura ambiente $T_{a m b}=500^{\circ} \mathrm{C}$.

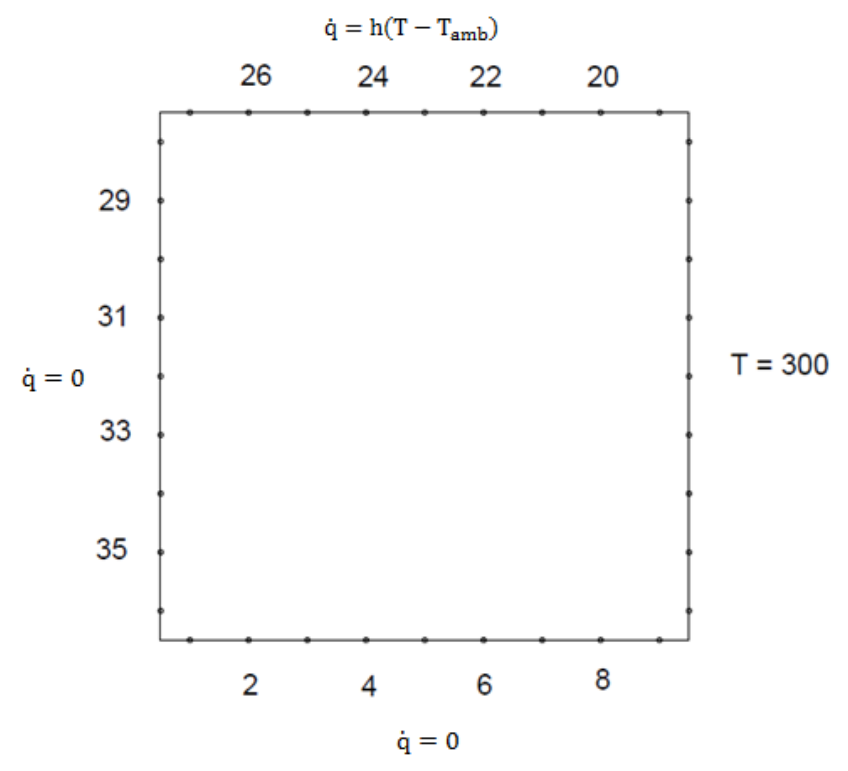

Figura 2. Geometria e condições de contorno do problema.

A Tabela 1 compara os resultados do programa criado com os presentes na referência acima citada. Como é observado, o erro máximo é de $0,07 \%$, o que dá a segurança de dizer que o código funciona para as três condições de contorno utilizadas.

Tabela 1. Comparação dos resultados da simulação 2D.

\begin{tabular}{c|c|c|c}
\hline Nó & $\begin{array}{c}\text { Temperatura } \\
\left({ }^{\circ} \mathrm{C}\right)\end{array}$ & $\begin{array}{c}\text { Temperatura } \\
\text { Programa }\left({ }^{\circ} \mathrm{C}\right)\end{array}$ & $\begin{array}{c}\text { Erro Relativo } \\
(\%)\end{array}$ \\
\hline 2 & 385,12 & 384,99 & 0,03 \\
\hline 4 & 372,98 & 372,93 & 0,01 \\
\hline 6 & 351,83 & 351,90 & 0,02 \\
\hline 8 & 323,48 & 323,72 & 0,07 \\
\hline 20 & 442,50 & 442,39 & 0,02 \\
\hline 22 & 469,57 & 469,45 & 0,03 \\
\hline 24 & 478,68 & 478,60 & 0,02 \\
\hline 26 & 482,30 & 482,23 & 0,01 \\
\hline 29 & 455,84 & 455,60 & 0,05 \\
\hline 31 & 424,88 & 424,71 & 0,04 \\
\hline 33 & 402,82 & 402,70 & 0,03 \\
\hline 35 & 390,57 & 390,46 & 0,03 \\
\hline
\end{tabular}

A Figura 3 mostra a distribuição de temperatura presente na solução. 


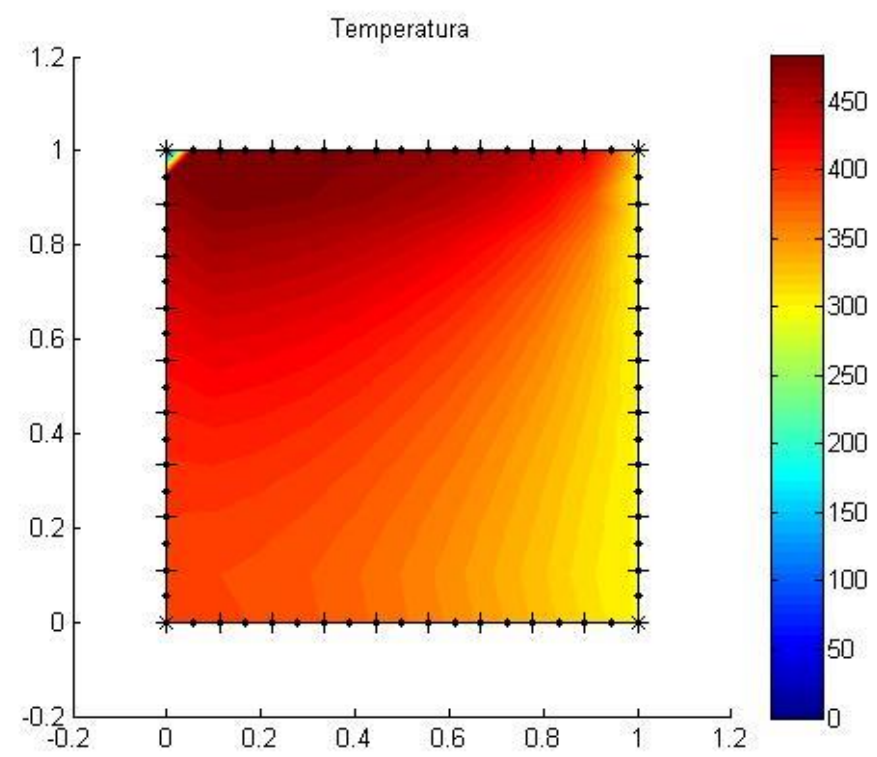

Figura 3. Distribuição de temperatura obtida na simulação permanente $2 D$.

\subsection{Programa Permanente 3D}

Ao implementar o método dos elementos de contorno em uma malha tridimensional foi simulado o mesmo problema representado pela Fig. 2 para validá-lo. Lembrando que, ao colocar duas superfícies opostas com fluxo de calor iguais a zero o problema se comporta como se fosse bidimensional. Os resultados da simulação se encontram na Tab. 2 .

Tabela 2. Comparação dos resultados da simulação $3 D$.

\begin{tabular}{c|c|c|c}
\hline Nó & $\begin{array}{c}\text { Temperatura } \\
\left({ }^{\circ} \mathrm{C}\right)\end{array}$ & $\begin{array}{c}\text { Temperatura } \\
\text { Programa }\left({ }^{\circ} \mathrm{C}\right)\end{array}$ & $\begin{array}{c}\text { Erro Relativo } \\
(\%)\end{array}$ \\
\hline 2 & 385,12 & 384,82 & 0,08 \\
\hline 4 & 372,98 & 372,70 & 0,07 \\
\hline 6 & 351,83 & 351,58 & 0,07 \\
\hline 8 & 323,48 & 323,29 & 0,06 \\
\hline 20 & 442,50 & 442,28 & 0,05 \\
\hline 22 & 469,57 & 469,48 & 0,02 \\
\hline 24 & 478,68 & 478,64 & 0,01 \\
\hline 26 & 482,30 & 482,25 & 0,01 \\
\hline 29 & 455,84 & 439,29 & 3,63 \\
\hline 31 & 424,88 & 412,47 & 2,92 \\
\hline 33 & 402,82 & 395,20 & 1,89 \\
\hline 35 & 390,57 & 387,79 & 0,71 \\
\hline
\end{tabular}

A Figura 4 nos mostra a distribuição de temperatura presente na solução. 


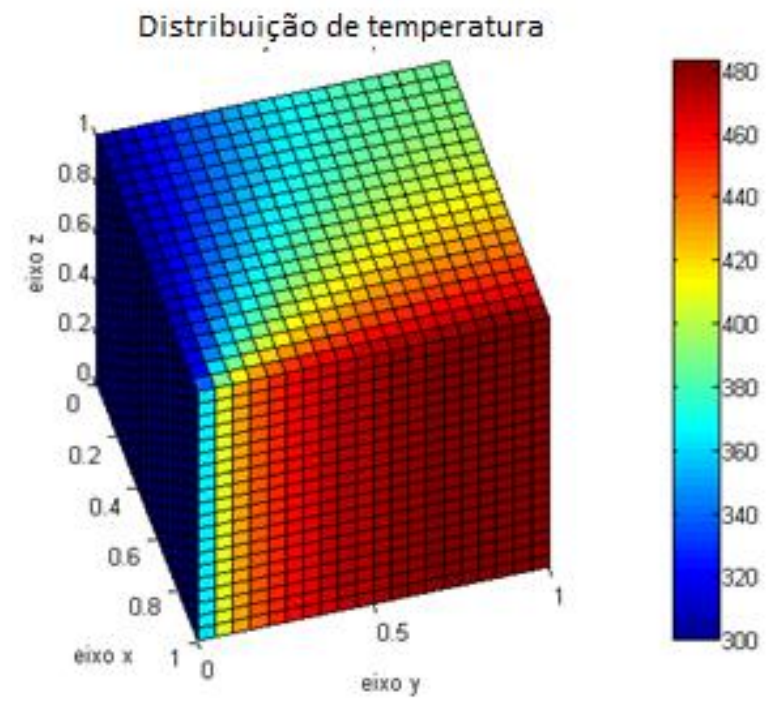

Figura 4. Distribuição de temperatura obtida na simulação permanente 3D.

Tanto para o programa bidimensional quanto para o programa tridimensional foram simulados casos com condição de contorno de radiação, como mostra a Fig. 5, as duas faces que não aparecem estão isoladas termicamente para que o problema se torne bidimensional.

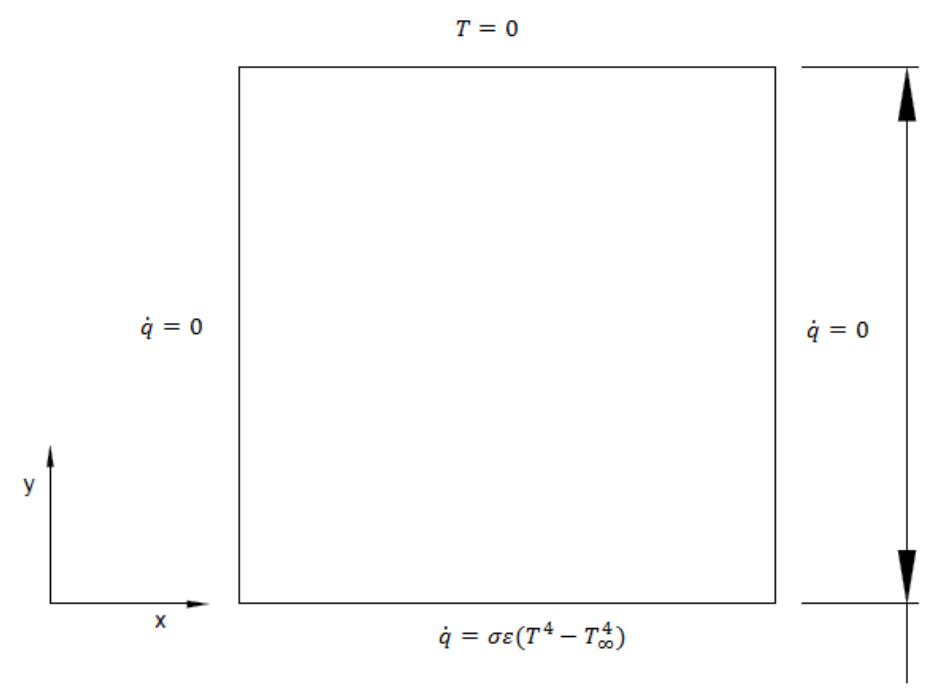

Figura 5. Geometria e condições de contorno do problema de radiação.

Onde a temperatura no infinito é igual a $100^{\circ} \mathrm{C}\left(T_{\infty}=100^{\circ} \mathrm{C}\right)$, a temperatura da face superior igual a $0^{\circ} \mathrm{C}\left(T=0{ }^{\circ} \mathrm{C}\right)$, as superfícies laterais são isoladas termicamente $\left(q=0 \mathrm{~W} / \mathrm{m}^{2}\right)$, emissividade do material igual a $1(\varepsilon=1)$, condutividade térmica do material igual a $1(k=1 \mathrm{~W} / \mathrm{m} . K)$ e constante de Stefan-Boltzmanm $(\sigma)$ igual a $5,67 \times$ $10^{-8} \mathrm{~W} / \mathrm{m}^{2} \mathrm{~K}^{4}$.

A comparação do resultado com a solução analítica do problema encontra-se na Tab. 3, e a distribuição de temperaturas na Fig. 6. Os erros são maiores quando feita a comparação com o valor analítico, chegando a 6,7\%, porém é um erro aceitável, validando assim o programa.

Tabela 3. Comparação dos resultados da simulação 3D da condição de contorno de radiação.

\begin{tabular}{c|c|c|c}
\hline Posição em y & $\begin{array}{c}\text { Temperatura } \\
\text { numérica }\left({ }^{\circ} \mathrm{C}\right)\end{array}$ & $\begin{array}{c}\text { Temperatura } \\
\text { analítica }\left({ }^{\circ} \mathrm{C}\right)\end{array}$ & $\begin{array}{c}\text { Erro Relativo } \\
(\%)\end{array}$ \\
\hline 0,05 & 5,33 & 5,39 & 0,97 \\
\hline 0,15 & 4,77 & 4,82 & 0,95 \\
\hline
\end{tabular}




\begin{tabular}{l|l|l|l}
\hline 0,25 & 4,21 & 4,25 & 0,97 \\
\hline 0,35 & 3,65 & 3,69 & 1,01 \\
\hline 0,45 & 3,09 & 3,12 & 1,06 \\
\hline 0,55 & 2,52 & 2,55 & 1,14 \\
\hline 0,65 & 1,96 & 1,98 & 1,27 \\
\hline 0,75 & 1,40 & 1,42 & 1,53 \\
\hline 0,85 & 0,83 & 0,85 & 2,22 \\
\hline 0,95 & 0,26 & 0,28 & 6,70 \\
\hline
\end{tabular}

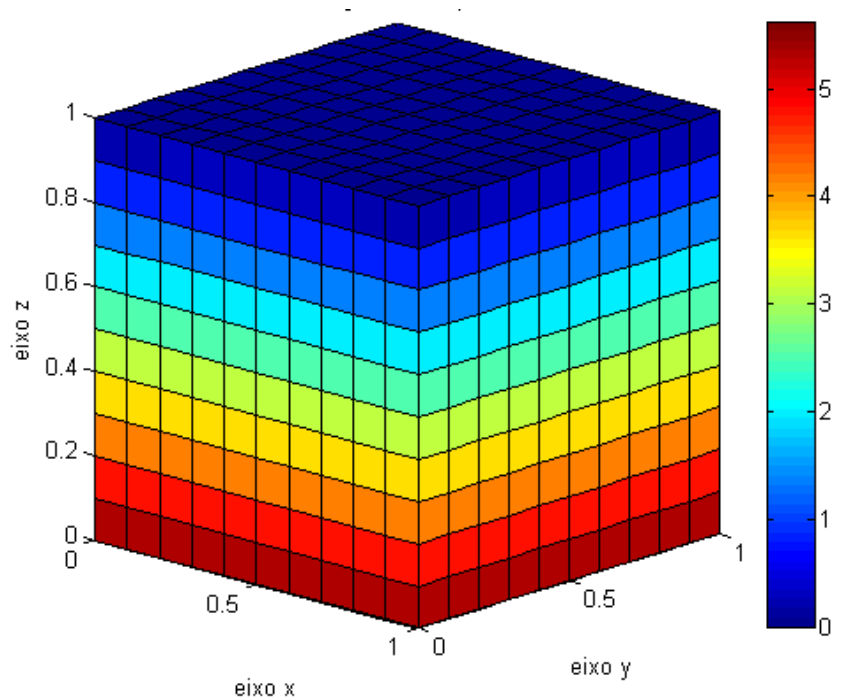

Figura 6. Distribuição de temperatura obtida na simulação permanente 3D de radiação.

\subsection{Programa Transiente 3D - Método da Reciprocidade Dual}

Mesmo o programa tendo sido revisado exaustivamente, algum erro ainda persiste. Para demonstrar o problema foi realizada uma simulação simples, um corpo quadrado de lado $10 \mathrm{~m}$ com temperatura inicial igual a $10^{\circ} \mathrm{C}$ sofre um choque térmico onde todas as suas faces ficam com uma temperatura de $0^{\circ} \mathrm{C}$. A Figura 7 mostra o comportamento da temperatura no ponto central feito por um programa que funciona para casos $2 \mathrm{D}$ com o método da reciprocidade dual.

Simulando o mesmo caso no programa 3D obtém-se a Fig. 8. O caso descrito acima foi um dos que obteve o erro mais sutil no ponto central, por isso foi utilizado para exemplificar o erro. Em todas as simulações algum ponto chega a temperaturas ou muito altas, ou muito baixas.

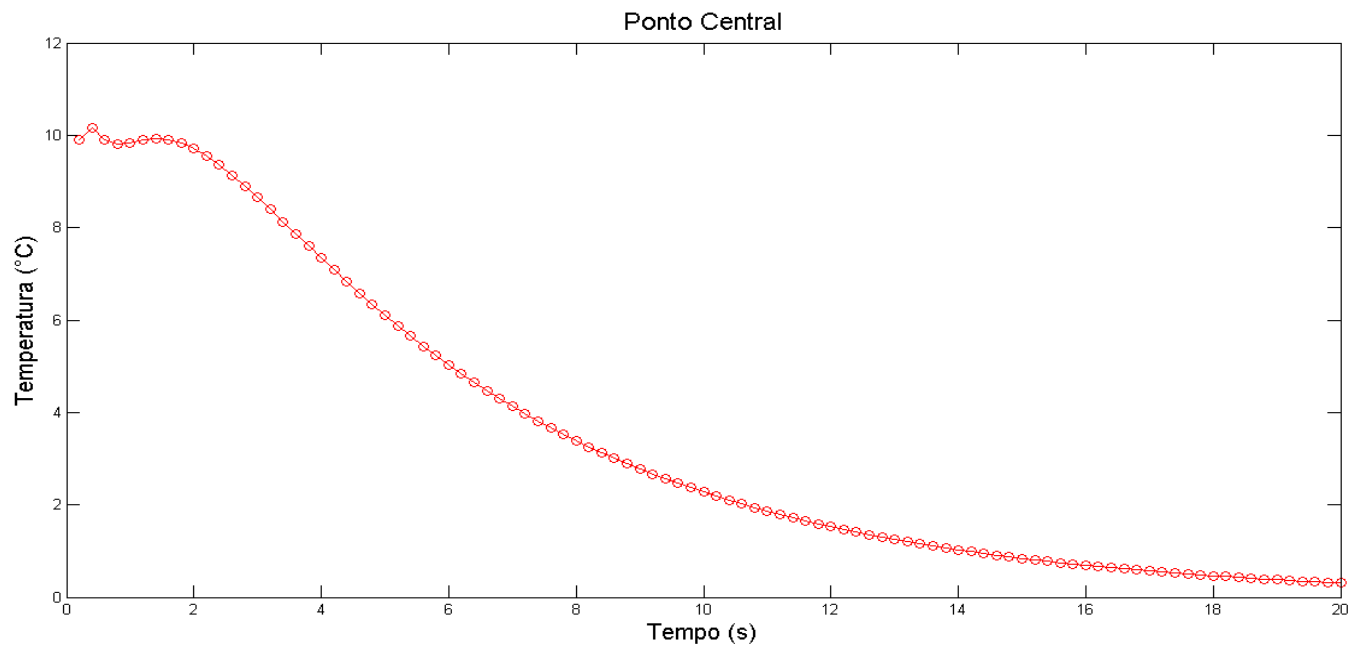

Figura 7. Distribuição de temperatura obtida na simulação permanente 3D de radiação. 


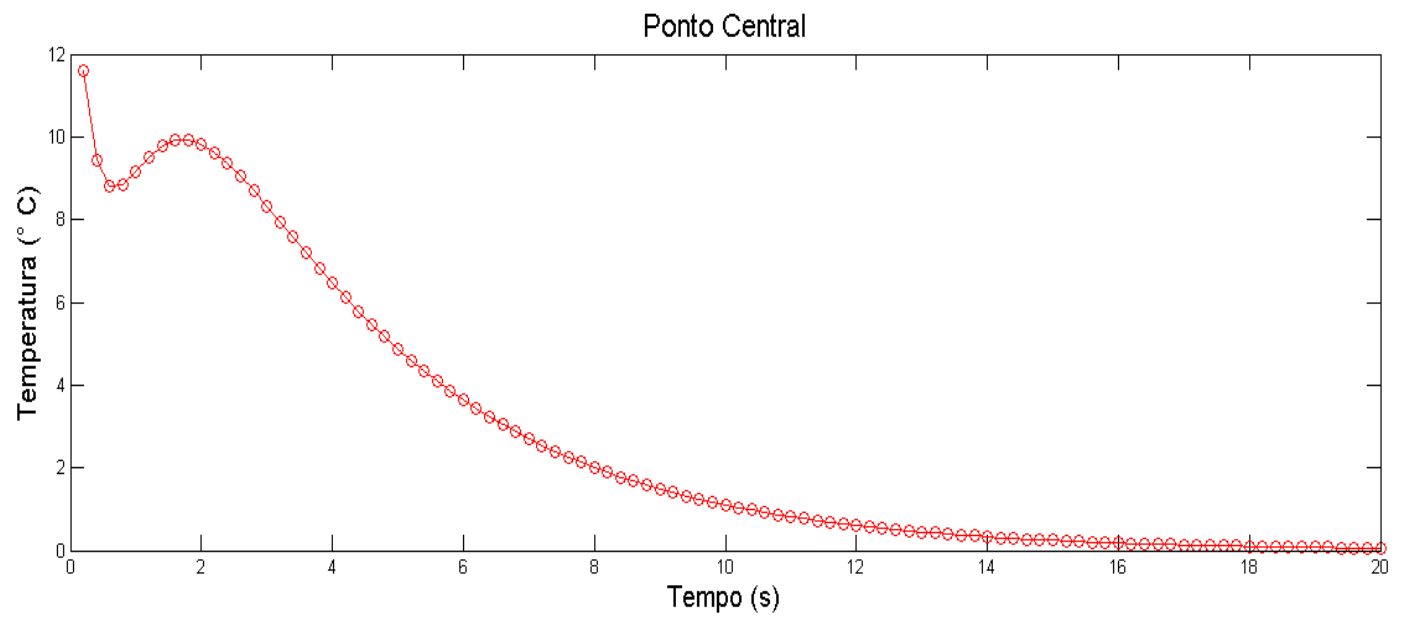

Figura 8. Distribuição de temperatura obtida na simulação permanente 3D de radiação.

Observando ambos os gráficos verifica-se que no início existe uma certa dificuldade de convergência em ambos os casos, porém com intensidades diferentes. A partir dos 2 segundos iniciais a temperatura se comporta como o esperado, indicando que o programa está correto até certo ponto.

Para solucionar o erro, foram verificados erros de cálculo, testadas outras funções de aproximação, uso de diferentes métodos para a integração no tempo e até testes com outras equações diferencias. No caso do uso de outras equações diferenciais, utilizou a equação de Helmholtz, onde foram obtidos erros maiores que o normal, porém aceitáveis. $\mathrm{O}$ programa em questão utiliza elementos constantes, que não varia a temperatura ao longo do elemento, portanto futuramente será feita a mesma simulação utilizando outro tipo de elemento para ver se o erro persiste.

\subsection{Programa Transiente 2D - Solução Fundamental Transiente}

Utilizando a solução fundamental transiente, foi criado um programa bidimensional capaz de simular as mesmas condições de contorno, para que num futuro seja desenvolvido também um programa tridimensional. O mesmo problema mostrado na Fig. 2 foi utilizado para um caso onde a temperatura já tenha se estabilizado. A distribuição de temperatura está representada na Fig. 9. O maior erro encontrado neste caso foi de 0,03\%, que não é significativo.

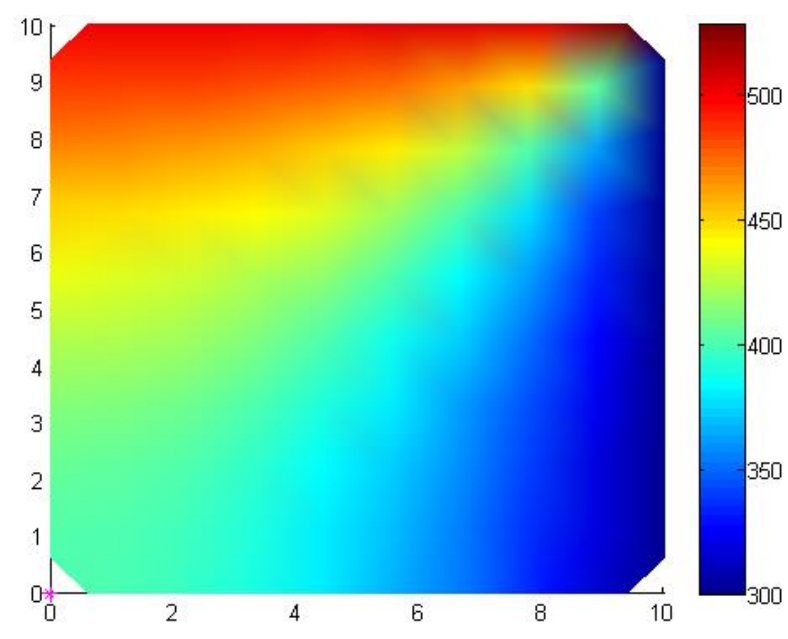

Figura 9. Distribuição de temperatura obtida na simulação transiente $2 \mathrm{D}$ com a solução fundamental transiente.

Para a condição de contorno de radiação, o programa não chega a uma solução estável. As temperaturas ficam variando pouco a pouco independente do período de tempo analisado. Esse erro é ocasionado por alguma aproximação feita pelo programa, já que os resultados se aproximam da resposta real.

\section{REFERÊNCIAS}

Brebbia, C. A., Dominguez, J. 1992. Boundary Elements. An Introductory Course, Southampton, United Kingdom :Ed. WIT Press, 
Effren, T. C. 1997. Implementação Computacional do Método dos Elementos de Contorno Para a Solução de Problemas de Difusão, Departamento de Engenharia Civil, COPPE/UFRJ, Rio de Janeiro, Brasil.

Kane, J. H. 1994. Boundary Elements Analysis in Engineering of Continuum Mechanics, New Jersey, United States: Ed. Prentice Hall.

Partridge, P. W., Brebbia, C. A., Wrobel, L. C. 1991. The Dual Reciprocity Boundary Elements Method, Southampton, United Kingdom: Ed. Computer Mechanics Publications e Elsevier Applied Science

\title{
RESPONSABILIDADE AUTORAL
}

O autor é o único responsável pelo conteúdo deste trabalho.

\section{TRANSIENTE HEAT TRANSFER SIMULATION USING THE BOUNDARY ELEMENTS METHOD}

\author{
Rafael Marques Campos, rmarquescampos@gmail.com
}

${ }^{1}$ Universidade de Brasília - UnB ... Campus Universitário Darcy Ribeiro, Faculdade de Tecnologia, Asa Norte, Brasília

\begin{abstract}
This work presents the development of a software for the purpose of numerically simulate the heat transfer in the rapid prototyping process which deposits successive layers of metal using arc welding, also known as $3 D$ welding. In order to able to predict the behavior of the deposited material, the heat transfer is analyzed. A numerical model using the boundary elements method is developed, from which it becomes possible to simulate certain conditions. The boundary conditions of temperature, heat flux, convection and radiation are considered, it is also possible to use punctual heat sources. Within the boundary element method two methods are used, the dual reciprocity method and the transient fundamental solution. At the end the code is validated by comparing the numerical results with analytical solutions.
\end{abstract}

Keywords: boundary element, heat transfer, dual reciprocity, fundamental solution 